\title{
Specific Regulation of TCP genes by miR319
}

\author{
Javier F. Palatnik ${ }^{1}$ and Detlef Weigel ${ }^{2}$ \\ ${ }^{1}$ Instituto de Biología Molecular y Celular de Rosario (IBR), Rosario, Argentina \\ 2 Max Planck Institute for Developmental Biology, Tübingen, Germany.
}

Contact information:

Javier Palatnik

palatnik@ibr-conicet.gov.ar

ORCID: https://orcid.org/0000-0001-7996-5224

Detlef Weigel

weigel@weigelworld.org

ORCID: https://orcid.org/0000-0002-2114-7963 


\begin{abstract}
MicroRNAs (miRNAs) are major regulators of gene expression in multicellular organisms. Many of the evolutionarily conserved miRNAs in plants are encoded by small gene families. The miR159/miR319 family has six members of similar sequences sharing 17 nucleotides in Arabidopsis thaliana. The members of this miRNA family regulate genes encoding TCP

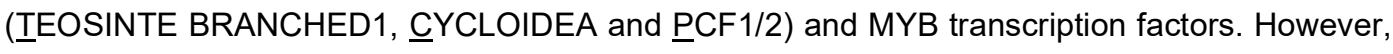
despite their sequence similarities, genetic evidence indicates that miR159 and miR319 fulfil different roles in vivo. Here, we confirm previous findings showing that TCP genes are not targeted by miR159. Thus, specific small sequence differences between the miRNAs allow for the specific regulation of TCP transcription factors by miR319 miRNAs.
\end{abstract}

\title{
Introduction
}

MiRNAs constitute a class of approximately 21 nucleotides long small RNAs that posttranscriptionally repress target genes in plants and animals. They are processed from longer precursors harboring a foldback structure with the miRNA embedded in one of its arms [reviewed in (Achkar et al., 2016; Yu et al., 2017)]. In plants, these precursors are processed in the nucleus by a complex formed by DICER-LIKE1 (DCL1) and accessory proteins such as HYPONASTIC LEAVES1 and SERRATE (Achkar et al., 2016; Yu et al., 2017). Mature miRNAs associate with ARGONAUTE (AGO) proteins, preferentially AGO1 in Arabidopsis [reviewed in (Ma and Zhang, 2018)]. The miRNAs guide AGO complexes to target RNAs by base complementarity, inhibiting their translation and/or guiding them to cleavage (Achkar et al., 2016; Yu et al., 2017). Plant miRNAs can recognize target RNAs with different degrees of complementarity, and the number and position of miRNA/target mismatches, the miRNA/target interaction energy and the context of the target sequence have been shown to affect miRNA-mediated gene regulation in plants (Allen et al., 2005; Li et al., 2014; Liu et al., 2014; Mallory et al., 2004; Schwab et al., 2005).

Many of the evolutionarily conserved miRNAs are encoded by small gene families, with the mature miRNAs being either identical or differing by a few nucleotides, but produced from the same precursor arm (Meyers et al., 2008). The Arabidopsis reference genome contains six genes encoding members of the miR159/miR319 family (Palatnik et al., 2007). All mature miRNAs are contained in the 3' arm of their respective precursors, which are processed by four sequential cuts by DCL1 (Bologna et al., 2009). Phylogenetic studies have suggested a common origin of all genes encoding members of the miR159/miR319 family (Li et al., 2011).

MiRNAs of the miR159/miR319 family show high sequence complementarity to several transcripts encoding transcription factors of the TCP and MYB class. Experimental evidence, however, indicates that members of this miRNA family fulfill different functions in vivo (Achard et al., 2004; Allen et al., 2007; Nag et al., 2009; Palatnik et al., 2003; Palatnik et al., 2007). 
Overexpression of miR319a or miR319b induces strong changes in leaf morphogenesis and crinkled leaves, which phenocopies higher order tcp mutants (Bresso et al., 2018; Efroni et al., 2008; Koyama et al., 2007; Koyama et al., 2010; Schommer et al., 2008). On the other hand, overexpression of miR159a or miR159b has no obvious effects on leaf morphology (Achard et al., 2004; Allen et al., 2007; Palatnik et al., 2007), and instead, results in stamen defects, similar to those seen in myb33 myb65 double mutants (Allen et al., 2007; Millar and Gubler, 2005).

Genetic evidence also highlights differences in the regulatory networks controlled by these miRNAs in Arabidopsis. The activation tagged mutant, jaw-D, is a miR319a overexpressor with crinkled leaves (Palatnik et al., 2003). A suppressor screen of jaw-D recovered mutations in the miR319-binding site of TCP4 (Palatnik et al., 2007). Furthermore, a point mutation in the sequence of mature miR319a results in narrow petals, which are reverted to wild-type shape by a compensatory mutation in TCP4 (Nag et al., 2009). On the other hand, mir159a mir159b double mutants have curled hyponastic leaves, similar to the phenotype of plants expressing a miR159resistant MYB33 transgene that has point mutations in the miRNA-binding site (Allen et al., 2007).

Furthermore, in mir159a mir159b myb33 myb65 quadruple mutants, in which two MIR159 genes and two of its $M Y B$ targets are simultaneously inactivated, near normal leaf shape is recovered, demonstrating that miR159a and miR159b are required in vivo to control the expression of genes encoding MYB transcription factors (Allen et al., 2007).

Our previous work showed that expression levels as well as sequence differences between miR159 and miR319 are essential for the selection of the target genes in vivo (Palatnik et al., 2007). As we realized that a duplicated figure panel had been incorporated in Figure 4B of that publication, we have repeated the entire experiment, and replicated our previous findings. Here, we present our confirmatory results, showing that miR159 miRNAs do not affect TCP4 mRNA or protein expression in transient in vivo assays.

\section{Results}

We analyzed publicly available small RNA-sequencing data (https://mpss.danforthcenter.org/) (Nakano et al., 2006) and determined the most abundant miRNA generated for each precursor of the miR159/miR319 family (Figure 1). The six mature miRNAs are identical at 17 of the 21 nucleotides (Figure 1). Flexibility in the processing of their precursors leads to a proportion of the mature miRNAs having slightly different lengths, or sequences being shifted by one or two nucleotides with respect to the most abundant small RNA generated from each precursor. 


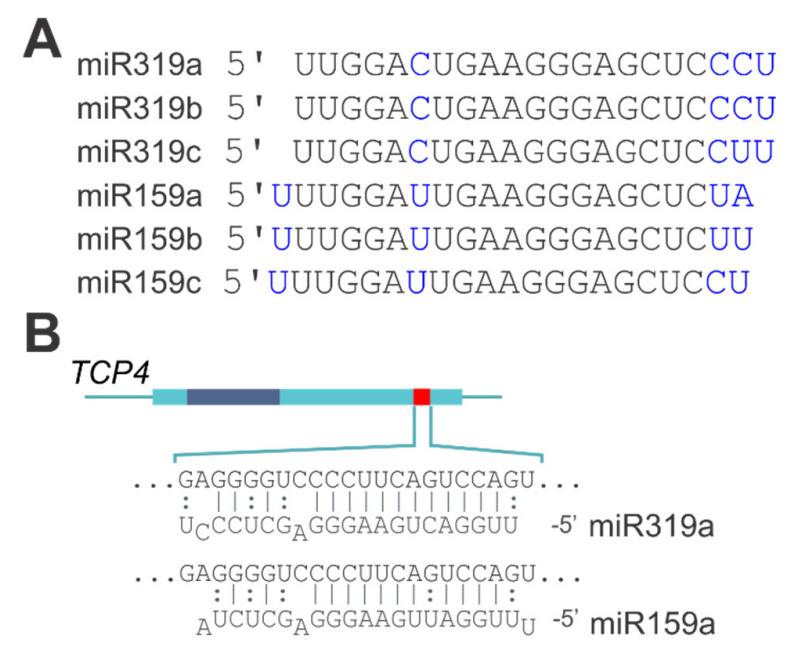

Figure 1. The miR159/miR319 family of miRNAs. (A) Sequences of the most abundant small RNA from each miRNA-encoding precursor. Blue letters highlight the differences. Note that miR159c accumulates to only very low levels. (B) Hybridization interface of TCP4 with miR319a and miR159a. The miRNA complementary region in TCP4 mRNA is highlighted as a red bar on top, and the region coding for the TCP DNA binding domain with a dark blue rectangle.

We analyzed with transient assays in Nicotiana benthamiana the effects of the different miRNAs on the accumulation of TCP4 transcript and protein (Figure 2). Expressing a TCP4:GFP transgene in $N$. benthamiana leaves allowed us to detect TCP4:GFP transcript (Figure 2A) and GFP fluorescence (Figure 2B), despite the presence of substantial levels of endogenous miR159 in $N$. benthamiana leaves (Figure 2A). Co-expression of miR319a led to strong reduction of both, TCP4:GFP transcript and GFP fluorescence (Figures 2A and 2B). Even the weaker expression of miR319b (which is identical in sequence to miR319a) from a 35S:miR319b transgene had a pronounced effect on TCP4:GFP RNA levels (Figure 2A). As a control, we used a version of TCP4:GFP with multiple mutations in the miRNA complementary motif (mTCP4), as described in Palatnik et al., 2003. mTCP4:GFP RNA levels appeared unchanged when miR319 was coexpressed, as expected for this miR319-insensitive version(Figure 2A).

Further increasing miR159 levels over the endogenous levels present in tobacco leaves by co-infiltrating 35S:miR159a or 35S:miR159b-encoding transgenes together with TCP4:GFP had no obvious effects on TCP4 mRNA levels or GFP fluorescence (Figure 2A and 2B). 
A

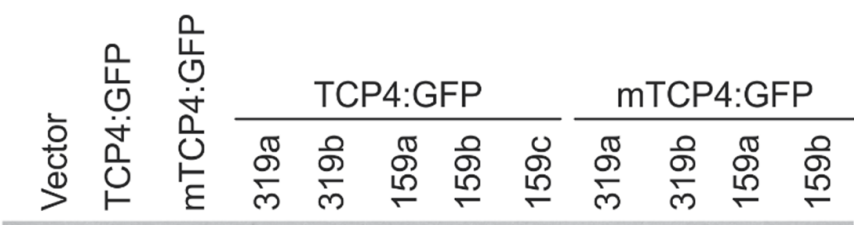
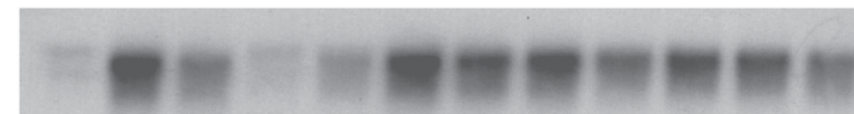

TCP4:GFP

$21 \mathrm{nt}$

$20 \mathrm{nt}-$

$\operatorname{miR} 159 a$

$21 \mathrm{nt}-$

$\operatorname{miR319a}$

$20 \mathrm{nt}^{-}$

B
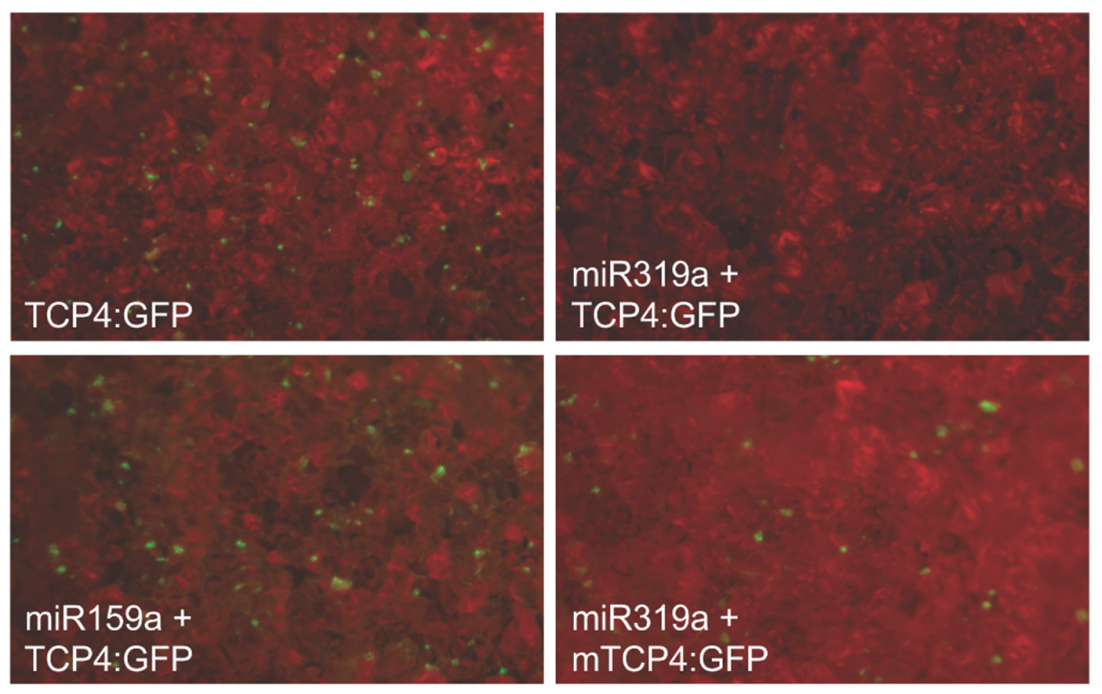

Figure 2. Specific regulation of TCPs by miR319. (A) Effect of different miRNAs on TCP4:GFP RNA levels in a N. benthamiana transient assay. (B) Effect of different miRNAs on TCP4:GFP fluorescence in $N$. benthamiana leaf epidermal cells.

\section{Conclusions}

Our results show that miR159 miRNAs do not regulate $T C P$ mRNA or protein accumulation, and support conclusions that specific sequence differences between miR159 and miR319 underlie the different in vivo functions of the two miRNAs (Palatnik et al., 2007). Previous data showed that both the G-U wobble at position 7 of miR159, as well as the mismatches at the miRNA 3' end prevent TCP4 from being targeted by miR159 (Palatnik et al., 2007). The evidence is also consistent with data that modifications in the miR319-binding site of TCP4 can compensate for changes in miR319 sequence (Nag et al., 2009) or levels (Palatnik et al., 2007). A similar situation 
is observed in the case of miR159 and MYB transcription factors, with mir159a mir159b mutant growth defects being suppressed by mutations in MYB33 and MYB65 (Allen et al., 2007).

\section{Material and Methods}

The experimental approaches were performed as described previously (Palatnik et al., 2007).

\section{Acknowledgements}

We thank Rebecca Schwab, Carla Schommer, Heike Wollmann, Ed Allen, Jim Carrington, Ramiro Rodriguez, Norman Warthmann and Jerôme Boisbouvier for comments on the manuscript and endorsing its content.

\section{Literature}

Achard, P., Herr, A., Baulcombe, D.C., and Harberd, N.P. (2004). Modulation of floral development by a gibberellin-regulated microRNA. Development 131, 3357-3365.

Achkar, N.P., Cambiagno, D.A., and Manavella, P.A. (2016). miRNA Biogenesis: A Dynamic Pathway. Trends Plant Sci 21, 1034-1044.

Allen, E., Xie, Z., Gustafson, A.M., and Carrington, J.C. (2005). microRNA-directed phasing during trans-acting siRNA biogenesis in plants. Cell 121, 207-221.

Allen, R.S., Li, J., Stahle, M.I., Dubroue, A., Gubler, F., and Millar, A.A. (2007). Genetic analysis reveals functional redundancy and the major target genes of the Arabidopsis miR159 family. Proc Natl Acad Sci U S A 104, 16371-16376.

Bologna, N.G., Mateos, J.L., Bresso, E.G., and Palatnik, J.F. (2009). A loop-to-base processing mechanism underlies the biogenesis of plant microRNAs miR319 and miR159. EMBO J 28, 3646-3656.

Bresso, E.G., Chorostecki, U., Rodriguez, R.E., Palatnik, J.F., and Schommer, C. (2018). Spatial Control of Gene Expression by miR319-Regulated TCP Transcription Factors in Leaf Development. Plant Physiol 176, 1694-1708.

Efroni, I., Blum, E., Goldshmidt, A., and Eshed, Y. (2008). A protracted and dynamic maturation schedule underlies Arabidopsis leaf development. Plant Cell 20, 2293-2306.

Koyama, T., Furutani, M., Tasaka, M., and Ohme-Takagi, M. (2007). TCP transcription factors control the morphology of shoot lateral organs via negative regulation of the expression of boundary-specific genes in Arabidopsis. Plant Cell 19, 473-484. 
Koyama, T., Mitsuda, N., Seki, M., Shinozaki, K., and Ohme-Takagi, M. (2010). TCP Transcription Factors Regulate the Activities of ASYMMETRIC LEAVES1 and miR164, as Well as the Auxin Response, during Differentiation of Leaves in Arabidopsis. Plant Cell 22, 3574-3588.

Li, J., Reichel, M., and Millar, A.A. (2014). Determinants beyond both complementarity and cleavage govern microR159 efficacy in Arabidopsis. PLoS Genet 10, e1004232.

Li, Y., Li, C., Ding, G., and Jin, Y. (2011). Evolution of MIR159/319 microRNA genes and their post-transcriptional regulatory link to siRNA pathways. BMC Evol Biol 11, 122.

Liu, Q., Wang, F., and Axtell, M.J. (2014). Analysis of complementarity requirements for plant microRNA targeting using a Nicotiana benthamiana quantitative transient assay. Plant Cell 26, 741-753.

Ma, Z., and Zhang, X. (2018). Actions of plant Argonautes: predictable or unpredictable? Curr Opin Plant Biol 45, 59-67.

Mallory, A.C., Reinhart, B.J., Jones-Rhoades, M.W., Tang, G., Zamore, P.D., Barton, M.K., and Bartel, D.P. (2004). MicroRNA control of PHABULOSA in leaf development: importance of pairing to the microRNA 5 ' region. Embo $\mathrm{J} 23,3356-3364$.

Meyers, B.C., Axtell, M.J., Bartel, B., Bartel, D.P., Baulcombe, D., Bowman, J.L., Cao, X., Carrington, J.C., Chen, X., Green, P.J., et al. (2008). Criteria for Annotation of Plant MicroRNAs. Plant Cell.

Millar, A.A., and Gubler, F. (2005). The Arabidopsis GAMYB-like genes, MYB33 and MYB65, are microRNA-regulated genes that redundantly facilitate anther development. Plant Cell 17, 705-721.

Nag, A., King, S., and Jack, T. (2009). miR319a targeting of TCP4 is critical for petal growth and development in Arabidopsis. Proc Natl Acad Sci U S A 106, 22534-22539.

Nakano, M., Nobuta, K., Vemaraju, K., Tej, S.S., Skogen, J.W., and Meyers, B.C. (2006). Plant MPSS databases: signature-based transcriptional resources for analyses of mRNA and small RNA. Nucleic Acids Res 34, D731-735.

Palatnik, J.F., Allen, E., Wu, X., Schommer, C., Schwab, R., Carrington, J.C., and Weigel, D. (2003). Control of leaf morphogenesis by microRNAs. Nature 425, 257-263.

Palatnik, J.F., Wollmann, H., Schommer, C., Schwab, R., Boisbouvier, J., Rodriguez, R., Warthmann, N., Allen, E., Dezulian, T., Huson, D., et al. (2007). Sequence and expression differences underlie functional specialization of Arabidopsis microRNAs miR159 and miR319. Dev Cell 13, 115-125. 
bioRxiv preprint doi: https://doi.org/10.1101/747790; this version posted August 28,2019 . The copyright holder for this preprint (which was not certified by peer review) is the author/funder. All rights reserved. No reuse allowed without permission.

Confirmatory Results Specific Regulation of TCP genes by miR319

Schommer, C., Palatnik, J.F., Aggarwal, P., Chetelat, A., Cubas, P., Farmer, E.E., Nath, U., and Weigel, D. (2008). Control of jasmonate biosynthesis and senescence by miR319 targets. PLoS Biol 6, e230.

Schwab, R., Palatnik, J.F., Riester, M., Schommer, C., Schmid, M., and Weigel, D. (2005). Specific effects of microRNAs on the plant transcriptome. Dev Cell 8, 517-527.

Yu, Y., Jia, T., and Chen, X. (2017). The 'how' and 'where' of plant microRNAs. New Phytol 216, 1002-1017. 